\title{
A Highly-Efficient and Compact Surface Plasmon Polaritons-based Long-Wavelength Cut-off Filter at Telecom Wavelengths
}

\author{
Seyed Morteza Ebadi (1) *, Student Member, OSA, and Jonas Örtegren (1) \\ Department of Natural Sciences, Mid Sweden University, SE-85170 Sundsvall, Sweden \\ *seyed-morteza.ebadi@miun.se
}

\begin{abstract}
This paper describes numerical results of an ultra-compact and efficient plasmonic cut-off filter based on a MIM waveguide. Simulation results confirm that by adjusting structural parameters, the desired spectral response can be easily obtained. (c) 2020 The Author(s)

OCIS codes: (240.6680) Surface plasmon; (130.7408) Wavelength filtering devices.
\end{abstract}

\section{Introduction}

Development of next generation of photonic integrated circuits (PICs) necessitates the integration of highly miniaturized and efficient optical components. In this regards, surface plasmon polaritons (SPPs) may substantially take part in this process. SPPs' distinctive features such as guide and confinement of light at nano-meter scale make plasmonic-based devices an excellent candidate [1-2]. The metal-insulator-metal (MIM) plasmonic waveguide attracts a great deal of attention, due to its strong mode confinement with justifiable level of loss and has been utilized in various applications. As a result, different types of optical devices based on MIM plasmonic waveguides such as switches [3], light absorbers [4], sensors [5] and so on are demonstrated. Among them, optical filters, due to their widespread applications, including wavelength-division multiplexing (WDM), imaging system, and fluorescence microscopy are of fundamental interest. However, despite the significant and important roles of optical filters in many applications, they might have been overlooked. Indeed, development of different types of optical filters may play a major part for realization of point-of-care (POC) diagnostic instruments and prompt detection of, for instance, infectious diseases, as well as in on-chip circuits, helping to achieve miniaturized optical components. Therefore, we have proposed a novel highly-compact and efficient tunable plasmonic long-wavelength cut-off filter at technologically significant optical channels.

\section{Design and Simulation Results of proposed Plasmonic Long-Wavelength Cut-off Filter}

Figure 1(a) displays the schematic configuration of proposed nanoplasmonic cut-off filter. The structure is made of two layers of silver, whose complex permittivity has been taken from tabulated data of Johnson and Christy [6]. The insulator is assumed to be Silicon dioxide. The following measures have been taken into account in order to achieve the numerical results in this letter. By carefully choosing the value of the waveguide width to be markedly smaller than that of wavelength of the incident light, only fundamental TM mode exists in the waveguide. The grid sizes are set to be $5 \mathrm{~nm} \times 5 \mathrm{~nm}$ along the $\mathrm{x}$ and $\mathrm{y}$ directions, respectively. Two power monitors $\mathrm{P}$ and $\mathrm{Q}$, respectively, are selected to be placed at the inlet and outlet of the waveguide to detect the incident and transmitted power. The transmission is determined as T=Pout/Pin. We have employed a commercial EM software tool, CST MWS, to attain the numerical results. We have utilized the simulation software with frequency domain solver that uses finite element method (FEM) to study the performance of the suggested structure. Figure 1 (b) exhibits the transmittance and reflectance of the cut-off filter for $\mathrm{W}=20 \mathrm{~nm}$, which indicates the width of the waveguide, $\ell=100 \mathrm{~nm}$ specifies the distance between the input port of the waveguide and the stepped-impedance resonators (SIR), which is also the distance between the two SIRs, $\mathrm{S}=90 \mathrm{~nm}, \mathrm{~d}=50 \mathrm{~nm}$, and $\mathrm{h}=30 \mathrm{~nm}$, respectively, represent the length, width and the height of the stepped impedance resonator. The cut-off wavelength is defined as the wavelength where the transmission is equal to $1 \%$ [7], which is $1279.3 \mathrm{~nm}$ in the proposed structure. 
(a)

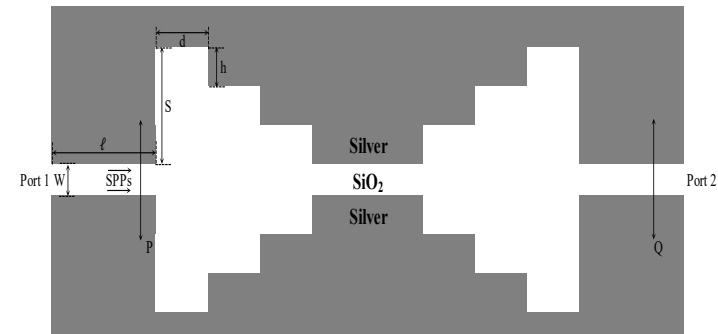

Fig. 1. (a) Schematic configuration of proposed long-wavelength cut-off (b) Transmission and reflection spectrum.

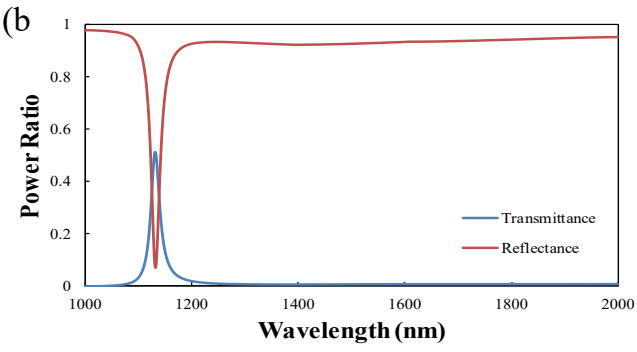

filter.

Figure 2 illustrates the transmission spectra of the plasmonic cut-off wavelength filter for various lengths, $\ell$, the distance between two SIRs, while all other parameters are kept the same as in Fig. 1(a). It is obvious that by altering the distance between the input port of the waveguide and the SIRs, one can easily adjust the cut-off wavelength. For instance, in the case of $\ell=85 \mathrm{~nm}$, the resonance wavelength is shifted towards shorter wavelength of $1111 \mathrm{~nm}$ from $1130.6 \mathrm{~nm}$ for $\ell=100 \mathrm{~nm}$, whereas for $\ell=130 \mathrm{~nm}$, the numerical results display in Fig. 2 that the cut-off wavelength is moved to longer wavelength of $1266.4 \mathrm{~nm}$. It is important to pointing out that adjusting the distance between the input waveguide port and the SIR structure, can also be used to modulate the cut-off wavelength. Furthermore, we have found that changing the width of the input waveguide port, $\mathrm{W}$, provides another way to adjust the resonance wavelength of the structure, which enables more degree of freedom when designing nano-meter scale optical circuits.

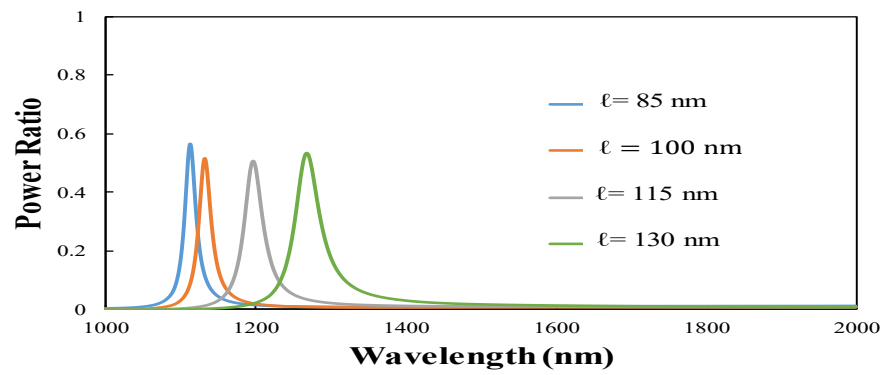

Fig. 2. The transmission profile of the suggested long wavelength cut-off filter with values defined in Fig.1 (a), as a function of $\ell$.

\section{Conclusion}

In conclusion, we have proposed a miniaturized and highly-efficient long-wavelength cut-off filter that operates at technologically important telecom wavelengths. The suggested plasmonic device is more efficient and compact compared with previous research studies [8,9], and may find applications in PICs and on-chip optical circuits.

\section{References}

[1] M. Kauranen and A. V. Zayats, "Nonlinear plasmonics," Nature photonics, vol. 6, no. 11, p. 737, 2012.

[2] Brongersma, Mark L., and Vladimir M. Shalaev. "The case for plasmonics." science 328.5977 (2010): 440-441.

[3] Khani, Shiva, Mohammad Danaie, and Pejman Rezaei. "All-Optical Plasmonic Switches Based on Asymmetric Directional Couplers Incorporating Bragg Gratings." Plasmonics (2019): 1-11.

[4] Chen, Yiting, et al. "Metal-insulator-metal plasmonic absorbers: influence of lattice." Optics express 22.25 (2014): 30807-30814.

[5] Gao, Huixuan, et al. "Ultraviolet broadband plasmonic absorber with dual visible and near-infrared narrow bands." JOSA A 36.2 (2019): $264-$ 269.

[6] P. B. Johnson and R.-W. Christy, "Optical constants of the noble metals," Physical review B, vol. 6, no. 12, p. 4370, 1972.

[7] Ebadi, Seyed Morteza, et al. "High-efficiency nanoplasmonic wavelength filters based on MIM waveguides." IEEE Photonics Technology Letters 28.22 (2016): 2605-2608.

[8] Ebadi, Seyed Morteza, et al, "A Multipurpose and Highly-Compact Plasmonic Filter Based on Metal-Insulator-Metal Waveguides," IEEE Photonics Journal 12.3 (2020): pp. 1-9.

[9] X. He, N. O’Keefe, D. Sun, Y. Liu, H. Uddin, A. Nirmalathas, and R. R. Unnithan, "Plasmonic Narrow Bandpass Filters Based on MetalDielectric-Metal for Multispectral Imaging," in CLEO Pacific Rim Conference 2018, OSA Technical Digest (Optical Society of America, 2018), paper Th4E.5. 